1561

STRATEGIES TO IMPROVE PAEDIATRIC INPATIENT PRESCRIBING PRACTICE IN THE UK

doi:10.1136/archdischild-2012-302724.1561

${ }^{1} \mathrm{~A}$ Whiteley, ${ }^{2} \mathrm{C}$ Pal, ${ }^{2} \mathrm{M}$ Woods. ${ }^{1}$ General Paediatrics; ${ }^{2}$ Paediatrics, Homerton University Hospital NHS Trust, London, UK

Background and Aims Errors are common in paediatric inpatient prescribing. This audit cycle assessed the impact of new strategies aimed at reducing prescribing errors.

Methods Drug charts from short-stay admissions in January 2010 were assessed retrospectively. Two gold standards were used; local hospital prescribing guidelines and the British National Formulary for Children (BNFC) 2009. From these results, a number of strategies were implemented to improve practice: prescribing training for new doctors was enhanced; a mandatory prescribing competency assessment was introduced; awareness was raised through local and regional presentations; increasing the availability of BNFC in clinical areas; daily pharmacy endorsements of drug charts and provision of Doctors' name stamps; and recommendations for a drug chart re-design. We then re-audited using identical methods in July 2010.

Results A total of 106 charts were assessed. Changes in documentation were found as follows:

Improvements: reason for non-administration (+26\%), allergy status $(+20 \%)$, at least one medication pharmacy-endorsed $(+16 \%)$, date of birth $(+5 \%)$, dose in $\mathrm{mg} / \mathrm{kg}$ where applicable $(+5 \%)$, frequency for all medications $(+2 \%)$, 'micrograms' written in full $(+1 \%)$.

No change: patient name (100\%), no unofficial abbreviations (100\%), weight (96\%), signature when administered (96\%).

Worsening: appropriate decimals $(-6 \%)$, doctor's name stamp $(-6 \%)$, start date $(-5 \%)$, total dose $(-2 \%)$, administration route $(-1 \%)$. Conclusions This audit revealed significant improvements in paediatric prescribing following implementation of our recommendations. However, key areas were identified for further improvement. Current work includes continued development of training, a dedicated prescribing area on the ward, regular monitoring of drug charts and continuing re-audit.

\section{MALTREATMENT IN CHILDHOOD AND ADOLESCENCE - HOW WE KNOW?}

doi:10.1136/archdischild-2012-302724.1562

'EO Veiga, 'SS Cordeiro, 'A Veiga, ${ }^{2} \mathrm{FM}$ Moliterno, ${ }^{2} \mathrm{NV}$ Moliterno, ${ }^{2} \mathrm{SA}$ Nogueira, ${ }^{2} \mathrm{~A}$ Siqueira, ${ }^{3} \mathrm{C}$ Cordebel. 'Paediatrics; 'Faculdade de Medicina de Petrópolis/HAC; ${ }^{3}$ Hospital Alcides Carneiro/FMP, Petrópolis, Brazil

Background and Aims Only with the 1988 Constitution (Article 227), Brazil began to recognize the child as "subject" of rights and not as property of their parents. This constitution created the Statute of Children and Adolescents (Law 8069/90) which states that children and adolescents are carrying special rights, ie they must be respected and protected. Today violence is considered a universal phenomenon that affects all ages, social classes, religions, and is one of the most serious public health problems, is the leading cause of infant mortality from the age of five. This study aimed to analyze the children admitted to the Teaching Hospital Alcides Carneiro, Brazil suspected of Maltreatment.

Methodology Cross-sectional study of cases reported to the Guardian Council of Petrópolis at the Pediatric Hospital Alcides Carneiro, from May 2007 to March 2010.

Results met the inclusion criteria of the study 61 patients, of whom $55 \%$ were female. Of the total, $15.27 \%$ had multiple types of abuse, a total of 72 reports $(63.7 \%$ Neglect, physical abuse $19.4 \%$, Sexual $12.7 \%$ and Psychological $4.2 \%$ ). As to age: infants 36\%, 13\% preschoolers, school $16.4 \%$ and $26.2 \%$ adolescents. The mother was responsible for $73.8 \%$ of assaults and father by $44.3 \%$, and in some cases both are equally liable.

Conclusion This study draws attention to the magnitude of the problem to be faced by those in the routine is responsible for the comprehensive care for the child's health.

\section{PEDIATRIC HOSPITALIZATIONS FOR IDENTIFICATION OF FOREIGN INJURIES TEACHING HOSPITAL IN THE PERIOD 2004 TO 2010}

doi:10.1136/archdischild-2012-302724.1563

'EO Veiga, ${ }^{2} \mathrm{~A}$ Veiga, ${ }^{2} \mathrm{SS}$ Cordeiro, 'SA Nogueira, 'NV Moliterno, ${ }^{2} \mathrm{FM}$ Moliterno. 'Paediatrics; ${ }^{2}$ Faculdade de Medicina de Petrópolis, Petrópolis, Brazil

Background and Aims In recent decades in our country, were deployed care programs and child health by facilitating the identification of external injuries. Among others, the disease control immunopreventable contributed to external causes was put among the leading causes of morbidity and mortality. The objective of this study was to identify pediatric hospitalizations for injuries in external Teaching Hospital, Maternal Child Health Reference of the city of Petrópolis, mountainous region of the State of Rio de Janeiro, Brazil.

Methods Cross-sectional study of pediatric hospitalizations with a diagnosis of Injury in the period 2004 to 2010 in Teaching Hospital is not referenced to childhood trauma.

Results 7397 children were hospitalized during the study period, 311 being distributed by external causes: TBI: 23.8\%, 17.4\% Burns; Mistreatment 17\%, 13.5\% exogenous poisoning; Multiple Trauma $8 \%$; Presence of foreign bodies in orifices natural $7.7 \%, 1.5 \%$ Semidrowning; Miscellaneous $11.1 \%$. The number cases/year in 2004 was $2.6 \%$ and $8.2 \%$ in 2010 . The gender distribution was $57.6 \%$ male, mean age of 5.2 years and duration of hospitalization was 7.3 days.

Conclusion The data analysis shows a relative increase of hospitalization for injuries in our hospital we attach to implementation and effectiveness of control programs for primary health care of the child.

\section{EFFECT OF AN INTERVENTION ON PERIPHERAL INTRAVENOUS CATHETER INFILTRATION RATES IN THE PEDIATRIC DEPARTMENT OF KING FAHAD ARMED FORCES HOSPITAL}

doi:10.1136/archdischild-2012-302724.1564

'M Abou Al-Seoud, ${ }^{1,2}$ MA Azzam, 'A Tanveer, ${ }^{1,2} \mathrm{D}$ El-Metwally, ${ }^{1} \mathrm{M}$ Al-Asnaj. 'Pediatrics, King Fahad Armed Forces Hospital, Jeddah, Saudi Arabia; 2Pediatrics and Neonatology, Suez Canal University (FOMSCU), Ismailia, Egypt

Background and Aims Peripheral intravenous catheterization is the most common invasive procedure among paediatric inpatients. Infiltration and extravasation are common complications, causing pain, discomfort, infection, nerve damage, and tissue necrosis. In an effort to reduce our rates, we designed a prospective intervention study.

Methods This was a prospective study performed in the Pediatric department of King Fahad Armed Forces Hospital. We recorded the infiltrations from Jan to June 2010, followed by the intervention during July and August 2010, in the form of in-services and laminated posters emphasizing site of catheterization, use of transparent tape and hourly cannula site inspection. The rates of infiltrations were then recorded from September 2010 - October 2011. Patients with any form of vasculitis, thrombotic or bleeding tendency were excluded. Descriptive data was recorded and rates were compared between pre- and post-intervention. Statistical analysis was performed using SPSS version 17.0. 
Results A total of 384 infiltrations were recorded. The average monthly rate pre-intervention was 26.8 and post-intervention was 20.7 with a p-value of 0.04 (statistically significant). Although a reduction was seen post-intervention, there was a rebound in infiltrations starting Nov 2010 to Feb 2011, which was recognized at the time, attributed to a high turnover of nurses and higher rates of admissions. We repeated a series of in-services with a subsequent reduction in infiltration rate.

Conclusions The overall pattern shows a statistically significant reduction in infiltration rate after the intervention. However, continuous surveillance and reinforcement of interventions is mandatory to sustain improved rates.

\section{USE OF THE MODIFIED BRIGHTON PEDIATRIC EARLY WARNING SCORE (PEWS) IN A NORWEGIAN DEPARTMENT FOR CHILDREN AND ADOLESCENTS}

doi:10.1136/archdischild-2012-302724.1565

EH Eggen, A Solevåg, J Schrøder, B Nakstad. Dept. of Pediatrics, Akershus University Hospital, Lørenskog, Norway

Background and Aims Structured observations and examination are crucial. However, paediatric early warning scoring systems is a relatively new concept.

We aimed to investigate the feasibility of a modified version of the Brighton paediatric early warning score (PEWS) in our department.

Methods The PEWS consists of respiratory, circulatory and behavioural parameters with $0-3$ points assigned for each category. Persisting postoperative vomiting and continuous inhalation medications give 2 extra points each. Hence, a score of 0 to13 can be assigned, score 0 being most favorable.

All acutely referred children in April/May 2011 were scored.

Patients were retrospectively categorized into diagnose groups. We included patients with score $0(n=89)$ and those with scores $\geq 4(n=49)$. Results The 0 group:

Sixty percent of patients considered to be well enough to be sent home without admittance to the ward had a PEWS of 0 . Only $10 \%$ of patients that were admitted had a PEWS of 0 . Allergic reactions (excl. anaphylaxis), arthritis, vasculitis, abnormal head circumference, psychosomatic disorders, constipation and upper respiratory tract infection typically gave low PEWS.

The $\geq 4$ group:

Diagnoses like asthma, bronchiolitis and other lower respiratory tract infections gave almost invariably high PEWS. In addition, cardiological conditions gave PEWS $\geq 4$.

PEWS scoring varied with age as 18/35 (60\%) of $0-2$ year olds scored $\geq 4,11 / 20(55 \%)$ at 2 years and only $17 / 83(20 \%)$ of patients $>3$ years scored $\geq 4$.

Conclusions The modified PEWS may be a useful method to detect high-risk patients in our department.

\section{PARENTS/CAREGIVERS' KNOWLEDGE TOWARDS MEDICINES ADMINISTRATION IN PAEDIATRICS}

doi:10.1136/archdischild-2012-302724.1566

C Teixeira de Barros, TM Salgado. iMed.UL - Research Institute for Medicines and Pharmaceutical Sciences, Faculty of Pharmacy, University of Lisbon, Lisbon, Portugal

Background and Aims The increasing complexity in paediatric patient care emphasizes patient safety as a topic of high priority. Parents/caregivers' lack of knowledge on how to administer extemporaneous formulations in paediatrics can be a potential source of medications errors.

Methods A 2-month cross sectional study was conducted with a convenience sample of paediatric outpatients' parents/caregivers from four hospitals in Lisbon. A questionnaire was developed to assess knowledge on how to administer the medicine (liquid or powder), how to measure the dose to be administered, administration schedule, storage conditions, validity period of the extemporaneous formulation, and adequate behaviour in case of missing a dose or vomiting immediately after taking the medicine. A univariate analysis was performed using SPSS v.19.

Results Eighty-four individuals participated in the study. The mean (SD) age was 34 (18.6) years, 26.0\% were non-Caucasian, $75.3 \%$ were married, $46.8 \%$ had an average of nine years of education and $50.6 \%$ were professionally active.

The mean level of knowledge as assessed by the questionnaire was $53.7 \%$. The lowest levels of knowledge were found for adequate behaviour in case of missing a dose or vomiting after taking the medicine, for which only $10.7 \%$ and $20.2 \%$ parents/caregivers, respectively, gave the correct answer. Non-Caucasian parents/caregivers and lower education level were significantly associated with a deficit of knowledge $(p<0.05)$.

Conclusions Low levels of knowledge were found among parents/ caregivers of paediatric patients. Strategies to enhance knowledge should be developed to improve patient safety.

\section{A REVIEW OF PAEDIATRIC HANDOVERS - ARE THEY SAFE AND EFFECTIVE?}

doi:10.1136/archdischild-2012-302724.1567

IP Nath, 'P Desai, ${ }^{2}$ W Kelsall. 'Neonatal Unit, Ipswich Hospital NHS Trust, Ipswich; ${ }^{2}$ Neonatal Unit, Cambridge University Hospitals NHS Foundation Trust, Cambridge, UK

Background With changing work patterns effective handovers are essential for patient safety and continuity of care. Handovers also provide educational opportunities, to initiate or complete work place based assessments (WPBA), improve communication, management skills to prioritise work.

Methods A 10 point online questionnaire was sent by email to all the trainees and tutors from June - August 2010.

Results Responses were received from 17 trusts (17/18 hospitals, 94\%) and from 56 trainees (18 ST1-3 and 38 ST4-8). Feedback was received on a total of 73 handovers: $36 \%$ covered both general paediatrics and neonates; $27 \%$ general paediatrics only and $23 \%$ neonatal intensive care. $78 \%$ of respondents were involved in two or more handovers during the working day. $69 \%$ of the handovers were lead by consultants or registrars. All the handovers had registrars present, $93 \%$ had junior trainees, $82 \%$ consultants and $34 \%$ had members from nursing team. $69 \%$ of the handovers were conducted with the aid of printed sheets. Only $51 \%$ of the handovers started on time, $19 \%$ were free from distractions by allied professionals and just $4 \%$ were 'bleep' free. $64 \%$ had some educational activity within the time allocated. WPBAs were initiated or completed in only $3 \%$ of handovers.

Conclusions Handover practice varies between hospitals. Most are well organised but many do not start on time and are not free from interruptions. Formal teaching was not a regular feature of handovers and WPBA were rarely initiated or completed. The role of the handover in training could be developed further.

\section{BURTON NEONATAL TRACK AND TRIGGER OBSERVATION CHART}

doi:10.1136/archdischild-2012-302724.1568

${ }^{1}$ M Ahmed, ${ }^{2}$ Phillips, ${ }^{1}$ A Manzoor. 'Paediatrics; ${ }^{2}$ Midwifery Sister, Burton Hospitals NHS Foundation Trust, Burton Upon Trent, UK

Background The use of early warning system scores and track \& trigger charts is widespread in adult and paediatric hospitalised patients. Its use in neonatal group is not well recognized. Lack of well established normal ranges for biophysical variables in preterm/ term neonates illustrate difficulties in establishing a scoring system 